\title{
On the Pitch-angle-dependent Perpendicular Diffusion Coefficients of Solar Energetic Protons in the Inner Heliosphere
}

\author{
N. Eugene Engelbrecht (10 \\ Center for Space Research, North-West University, Potchefstroom, 2522, South Africa; n.eugene.engelbrecht@gmail.com \\ Received 2019 May 7; revised 2019 June 5; accepted 2019 June 7; published 2019 July 25
}

\begin{abstract}
Various numerical solar energetic particle (SEP) transport studies have shown that perpendicular diffusion plays a significant role in the propagation of these particles in the heliosphere. In particular, computed SEP intensities and anisotropies have been shown to be sensitive to the pitch-angle dependence of the perpendicular diffusion coefficient as well as its magnitude. This study proposes a novel approach to the calculation of this quantity and compares this to the results of previous theoretical approaches. These various perpendicular diffusion coefficient expressions are demonstrated for turbulence conditions prevalent at Earth and closer to the Sun.
\end{abstract}

Key words: diffusion - solar energetic particles - turbulence

\section{Introduction}

A growing body of evidence, both observational (see, e.g., McKibben et al. 2001; Zhang et al. 2003; Dresing et al. 2012) and computational/theoretical (see, e.g., Zhang et al. 2009; Droge et al. 2010; He 2015; Strauss et al. 2017) supports the idea that perpendicular diffusion plays a significant role in the transport of solar energetic particles (SEPs) in the heliosphere. Due to the significance of pitch-angle scattering in the transport of SEPs, many studies focus on the pitch-angle diffusion coefficient $D_{\mu \mu}$ and its role in their diffusion parallel to some mean field (e.g., Karimabadi et al. 1992; Reames 1999; Shalchi 2009; He \& Wan 2012; Agueda \& Vainio 2013). To date, however, fewer studies have focused on the pitch-angledependent Fokker-Planck perpendicular diffusion coefficient $D_{\perp}$ (see, however, Qin \& Shalchi 2014; Wang et al. 2017), which enters directly into the Skilling (1971) equation solved in computational studies of SEP transport and, thus, could potentially play a crucial role in studies of space weather (e.g., Schwadron et al. 2014; Laitinen et al. 2018). These particle transport simulations, however, have shown that calculated SEP intensities and anisotropies are sensitive to the magnitude as well as the pitch-angle dependence of this quantity (Zhang et al. 2009; Dresing et al. 2012; Laitinen et al. 2013, 2018; Strauss \& Fichtner 2015; Strauss et al. 2017). Furthermore, it has been shown that particle transport across magnetic discontinuities is also sensitive to the pitch-angle dependence of $D_{\perp}$ (e.g., Strauss \& Fichtner 2014, 2015; Strauss et al. 2016).

Several approaches to calculating $D_{\perp}$ have been taken in various computational studies, and several theoretical models have been proposed. Earlier studies assumed a constant value for this quantity (Zhang et al. 2009) or a parameterized scaling of $D_{\perp} \sim \sqrt{1-\mu^{2}}$ (Droge et al. 2010; Dresing et al. 2012; where $\mu$ denotes the cosine of the particle pitch angle). The Jokipii (1966) field line random walk (FLRW) result, where it is assumed that turbulence levels are low and that particle Larmor radii are small relative to the turbulent correlation scale, has been used with some success in numerical SEP transport models to reproduce observed electron intensities and anisotropies (Strauss et al. 2017), probably due to the fact that these particles would naturally satisfy the assumptions implicit to this approach due to their small Larmor radii (see also Bain et al.
2011). Given the contextual predictions for turbulence conditions made by Chhiber et al. (2019), the ratio of the Larmor radius to the turbulence correlation scale should still be small even for SEP protons. However, Chhiber et al. (2019) also show, using a turbulence transport model, that turbulence levels are expected to rise steeply as the distance to the Sun decreases, which would possibly render the assumption of an FLRW approach for describing the perpendicular diffusion of these particles questionable, for higher energy protons at least. This would suggest that an alternate approach, such as that derived by Qin \& Shalchi (2014) from unified nonlinear theory (UNLT) of Shalchi (2010) for the case where pitch-angle scattering dominates the transport of these particles or that resulting from the approach taken by Fraschetti (2016), who derives a coefficient based solely on the mechanisms of gradient and curvature drift that scales as $\sim\left(\left(1-\mu^{2}\right) / 2\right)^{2}$. It is unclear, however, if perpendicular transport can rely solely on these mechanisms, given the influence of turbulence on particle drifts (see, e.g., Minnie et al. 2007b; Tautz \& Shalchi 2012; Engelbrecht et al. 2017). Furthermore, Webb et al. (2001) evaluate the Bhatnagar-Gross-Krook (BGK) Boltzmann equation and find that $D_{\perp} \sim\left(1-\mu^{2}\right)$. The various approaches to the calculation of $D_{\perp}$ mentioned above yield significantly different values for this quantity, which would significantly affect numerical SEP transport code results (e.g., Strauss et al. 2017) and display very different $\mu$ dependences, which would also have a significant effect on model results (Strauss \& Fichtner 2015). Notably, the FLRW result predicts that $D_{\perp} \sim|\mu|$, while the pitch-angle-scattering-dominated UNLT result predicts that $D_{\perp} \sim \mu^{2}$.

Direct numerical test particle simulations of diffusion coefficients in realistic simulated turbulence conditions can prove useful in studying the cross-field diffusion coefficients of SEPs. Relatively few such studies for $D_{\perp}$ have been published, most notably those by Casse et al. (2002), done for simulated inertial range slab turbulence, and those of Qin \& Shalchi (2014), for more realistic composite turbulence (see also Laitinen \& Dalla 2017). For low levels of turbulence, and small ratios of the particle Larmor radius to turbulence correlation scale, the FLRW approach yields perpendicular diffusion coefficients in good agreement with simulations (Qin \& Shalchi 2014), while for higher ratios of Larmor radius to 
correlation scale displays a pitch-angle cosine dependence similar to that expected from the UNLT of Shalchi (2010) when pitch-angle scattering dominates particle transport. It should be noted, however, that none of the abovementioned scalings for $D_{\perp}$ with $\mu$ reproduces all the features seen from simulations, which will be discussed in more detail below.

The aim, then, of this study is to provide additional insight for the SEP transport modeling community into the various results of the differing theoretical approaches taken to calculate $D_{\perp}$ and is achieved in a twofold manner. First, we propose an alternative approach to calculating $D_{\perp}$ based on the random ballistic decorrelation interpretation of the nonlinear guiding center (NLGC) theory proposed by Ruffolo et al. (2012), taking into account moderate levels of turbulence as are expected from spacecraft observations and turbulence transport modeling in the very inner heliosphere. This theory is chosen in part due to the fact that it provides an explicit expression for the perpendicular diffusion coefficient, as opposed to the implicit expressions yielded by the standard NLGC or variants thereof (see, e.g., Shalchi 2009). An expression for $D_{\perp}$ is calculated using this approach assuming an observationally motivated form for the turbulence power spectrum. Furthermore, given the mathematical complexity of this expression, an analytical approximation based on the NLGC theory result derived by Shalchi et al. (2004) is also presented. Second, we provide some insight into the differences between the various theoretical expressions derived in the abovementioned studies as well as those derived here, by making comparisons between these expressions, as evaluated both for typical solar minimum turbulence conditions at $1 \mathrm{au}$, and in the very inner heliosphere, assuming radial scalings for basic turbulence quantities motivated by the simulation results of Chhiber et al. (2019). Although the exact details of the behavior of turbulence in these regions are expected to remain the subject of much debate until the Parker Solar Probe observations provide some clarity (see, e.g., Zank et al. 2018; Adhikari et al. 2019), such an approach can provide insight into possible particle transport conditions, as has previously been shown by Engelbrecht \& Burger (2013a), Wiengarten et al. (2016), and Chhiber et al. (2017).

\section{Perpendicular Diffusion}

As a point of departure, the Taylor (1922)-Green (1951)Kubo (1957) expression that is fundamental to many scattering theories (see, e.g., Shalchi 2009) is employed. This approach is also often used as the basis for calculating diffusion coefficients from numerical test particle simulations of particle transport in various turbulence scenarios (e.g., Casse et al. 2002; Qin et al. 2002; Minnie et al. 2007a; Tautz \& Shalchi 2012; Riordan \& Pe'er 2019) and is given by

$$
D_{i i}=\int_{0}^{\infty}\left\langle v_{i}(0) v_{i}(t)\right\rangle d t
$$

where subscript $i$ denotes an axis of some Cartesian coordinate system, $v$ is the component of the particle velocity along said axis, and the angle brackets an ensemble-averaging process. For an alternative approach of incorporating the effects of adiabatic focusing, see Wang et al. (2017). In this study, a coordinate system with the $z$-axis along the uniform component $B_{o}$ of a magnetic field, $\boldsymbol{B}$, with an axisymmetric, transverse fluctuating component $\boldsymbol{b}$ such that $\langle B\rangle=B_{o}$ is assumed.
Ideally, an exact expression for the velocity components should be employed in Equation (1). Fraschetti \& Jokipii (2011) and Fraschetti (2016) employ an expression for the perpendicular guiding center velocity derived by Rossi \& Olbert (1970) that is accurate to the first order in $b_{x} / B_{o}$ and is gyroperiod-averaged, given in terms of the cosine of the particle pitch-angle $\mu$ as

$$
\tilde{\boldsymbol{v}}_{\perp}=\frac{v p c}{Z e B^{3}}\left[\frac{1-\mu^{2}}{2} \boldsymbol{B} \times \nabla B+\mu^{2} B(\nabla \times \boldsymbol{B})_{\perp}\right],
$$

with $Z$ as the atomic number, $e$ as the electron charge, $p$ as the particle momentum, and $c$ as the speed of light. Note that the tilde is used here to denote velocities corresponding to particle gyrocenters. An analytical evaluation of even this expression for the purpose of incorporation into Equation (1) is difficult (see, e.g., Fraschetti \& Jokipii 2011), and it is difficult to glean a clear $\mu$ dependence from these results that can be easily compared to the results of numerical test particle simulations, such as those reported by Qin \& Shalchi (2014). Considering the $\mu$ dependence of $\tilde{v}_{\perp}(0) \tilde{v}_{\perp}(t)$, however, it is clear that such a dependence would be a fourth-order polynomial, with no oddpowered terms, and, hence, an even function of $\mu$ as suggested by the simulations of Qin \& Shalchi (2014). Therefore, to avoid the complications implicit to the use of Equation (2), the $x$ and $y$ components of the speed of a particle's gyrocenter are expressed as

$$
\tilde{v}_{x}^{2}=\tilde{v}_{y}^{2}=a^{2} v_{z}^{2} \frac{b_{x}^{2}}{B_{o}^{2}}
$$

following rather the approximate approach of, e.g., Matthaeus et al. (2003) and Ruffolo et al. (2012), with the constant $a$ being a measure of how much particles "stick" to field lines (see also Shalchi 2009). Given that $v_{z}=\mu v$ with $v$, the particle speed, Equation (1) becomes

$$
D_{x x}=\frac{a^{2} v^{2}}{B_{o}^{2}} \int_{0}^{\infty}\left\langle\mu(0) \mu(t) b_{x}(0) b_{x}(t)\right\rangle d t .
$$

Following Matthaeus et al. (2003) in the assumption that particle velocities are not correlated with the local magnetic field vector, the above becomes

$$
D_{x x}=\frac{a^{2} v^{2}}{B_{o}^{2}} \int_{0}^{\infty}\langle\mu(0) \mu(t)\rangle\left\langle b_{x}(0) b_{x}(t)\right\rangle d t,
$$

where the magnetic autocorrelation function can now be dealt with following the various approaches of, e.g., Matthaeus et al. (2003), Shalchi (2010), or Ruffolo et al. (2012), who relate the magnetic correlation function to the Eulerian correlation function, $R_{x x}$, using Corrsin's independence hypothesis (see, e.g., Tautz \& Shalchi 2010), so that

$$
D_{x x}=\frac{a^{2} v^{2}}{B_{o}^{2}} \int_{0}^{\infty}\langle\mu(0) \mu(t)\rangle\left[\int R_{x x}(\boldsymbol{y}, t) P(\boldsymbol{y} \mid t) d^{3} y\right] d t,
$$

where here, as in Matthaeus et al. (2003), $P(\boldsymbol{y} \mid t)$ is the probability density of the particle experiencing a displacement, $\boldsymbol{y}$, at some $t$.

The $\mu$ dependence of the perpendicular diffusion coefficient derived using this approach will lie in that of the pitch-angle 
cosine autocorrelation function. Some guidance may be found in numerical test particle simulations. Casse et al. (2002) present simulations of this quantity for inertial range slab turbulence, showing that $\langle\mu(0) \mu(t)\rangle \sim \mu^{2} f(t)$, where $f(t)$ is an exponentially decreasing function of time. Qin \& Shalchi (2009) also report on direct results of simulations of the pitchangle cosine autocorrelation function for various levels of composite slab/2D turbulence and find that for low levels of turbulence $(\delta B / B=0.01), \mu$ remains approximately constant, while for higher levels of turbulence $(\delta B / B=1.0)$, the autocorrelation function displays strong fluctuations. At intermediate levels closer to what is observed at $1 \mathrm{au}(\delta B /$ $B=0.1)$, the autocorrelation function behaves similarly to what is reported by Casse et al. (2002). The simulations of $D_{\perp}$ reported on by Qin \& Shalchi (2014) were calculated for composite turbulence more representative of solar wind conditions at Earth (see, e.g., Bieber et al. 1994) and show that $D_{\perp}$ assumes finite values at $\mu=0$. Furthermore, these values appear to increase as test particle energies were increased. Strauss \& Fichtner (2014), from their simulations of particle intensities as observed by the Voyager spacecraft near the heliopause, expect a peak in $D_{\perp}$ at $90^{\circ}$ pitch, but turbulence in this region is very different (see, e.g., Burlaga et al. 2018) to the conditions assumed in the simulations of Qin \& Shalchi (2014).

The behavior of $D_{\perp}$ reported by Qin \& Shalchi (2014) when $\mu=0$ could imply that the $\mu$ autocorrelation function may not be a function of $\mu^{2}$ alone, which may be related to the effects of 2D turbulence (see, e.g., Karimabadi et al. 1992). At values of $\mu$ closer to \pm 1 , the perpendicular diffusion coefficient displayed a $\mu^{2}$ dependence with a slope varying with energy, and, at the lowest energies, a $\mu^{2}$ dependence in agreement with the FLRW prediction, assuming that $a^{2}=1 / 3$. Note, however, that the pitch-angle-scattering-dominated result from the UNLT as well as the FLRW result predicts a zero value for $D_{\perp}$ when $\mu$ is zero. Lastly, the simulations of Qin \& Shalchi (2014) show that $D_{\perp}$ is symmetric about the axis defined by $\mu=0$, as would be expected from Equation (2). The simulation results for the $\mu$ autocorrelation function presented by Qin \& Shalchi (2009), however, do not provide information about alternate pitchangle dependences beyond that reported by Casse et al. (2002), so as a first approach, it is assumed that

$$
\langle\mu(0) \mu(\tau)\rangle \sim \mu^{2} \exp (-t / \tau)
$$

assuming, due to the fact that $|\mu| \leqslant 1$, that higher order terms in $\mu$ are negligible. This would imply that $D_{\perp}=0$ when $\mu$ is zero but serves as a reasonable point of departure given existing simulation results for the $\mu$ autocorrelation function. The pitchangle cosine autocorrelation function is assumed to decrease exponentially with time, with $\tau$ some decorrelation timescale, following an approach similar to that of, e.g., Matthaeus et al. (2003) and motivated by the simulation results of Casse et al. (2002). The rest of Equation (5) is treated following the random ballistic decorrelation approach of Ruffolo et al. (2012). Those authors assume that guiding center displacement probability distributions are both Gaussian (see Lasuik \& Shalchi 2018 for an analysis of this assumption) and independent in each direction, as well as writing the correlation function, $R_{x x}$, as the Fourier transform of the 2D modal turbulence power spectrum, $S_{x x}(\boldsymbol{k}) e^{-\gamma(\boldsymbol{k}) t}$ (see Matthaeus et al. 2007), so that Equation (5) becomes

$$
D_{x x}=\frac{a^{2} v^{2}}{B_{o}^{2}} \mu^{2} \int S_{x x}(\boldsymbol{k}) T(\boldsymbol{k}) d \boldsymbol{k},
$$

with $\gamma$ as a dynamical decay rate (see Bieber et al. 1994; Gammon \& Shalchi 2017) and, with the backtracking correction proposed by Ruffolo et al. (2012),

$$
T(\boldsymbol{k})=\sqrt{\frac{\pi}{2 \sum_{i} k_{i}^{2} \tilde{v}_{i}^{2}}} \operatorname{erfc}\left(\frac{\tau^{-1}+\gamma(\boldsymbol{k})}{\sqrt{2 \sum_{i} k_{i}^{2} \tilde{v}_{i}^{2}}}\right) .
$$

The spectrum, $S_{x x}$, is assumed to be that corresponding to 2D fluctuations only, motivated by the simulations of Qin et al. (2002; see also Shalchi 2006). Equation (8) can, given some assumed form for $S_{x x}$, be directly evaluated in much the same way as was done by Wiengarten et al. (2016) for the pitchangle isotropic case.

The behavior of $D_{\perp}$ at $\mu=0$ reported by Qin \& Shalchi (2014) can, in part, be modeled if it is assumed that the pitchangle dependence of the autocorrelation function is somewhat different to that of Equation (7), so that

$$
\langle\mu(0) \mu(\tau)\rangle \sim\left(\alpha \mu^{2}+\beta\right) \exp (-t / \tau)
$$

assuming again that higher order terms in $\mu$ are negligible and that both $\alpha$ and $\beta$ are positive and constant. This approach is similar to that taken to correct for possible nonlinear effects in the calculation of the pitch-angle diffusion coefficient, $D_{\mu \mu}$, by Droge et al. (2010). Then

$$
D_{x x}=\frac{a^{2} v^{2}}{B_{o}^{2}}\left(\alpha \mu^{2}+\beta\right) \int S_{x x}(\boldsymbol{k}) T(\boldsymbol{k}) d \boldsymbol{k},
$$

but it is not entirely clear what choices need to be made to the constants $\alpha$ and $\beta$, which could possibly be determined from numerical simulations. In the following section, expressions for $D_{\perp}$ will be presented, derived from Equations (8) and (11) for an observationally motivated form for the 2D turbulence power spectrum. These expressions will then be compared with the FLRW and UNLT results presented by Qin \& Shalchi (2014).

\section{Perpendicular Diffusion Coefficients Compared}

To evaluate Equation (8) (and also Equation (11)), several assumptions need to be made. The decorrelation rate for the $\mu$ autocorrelation function needs to be modeled. One alternative is the approach taken by Matthaeus et al. (2003), where it is assumed that $\tau^{-1}=v / \lambda_{\|}$. It is not necessarily clear whether this approach would be suitable for $\mu$, however. Another candidate would be to assume that $\tau^{-1}=D_{\mu \mu}$. Both cases will be considered here. In what follows, the dynamical decay rate is set to zero (the magnetostatic case). This is a reasonable assumption, unless low-energy electrons are considered (for more details, see Engelbrecht \& Burger 2013b; Gammon \& Shalchi 2017; Strauss \& le Roux 2019).

As an axisymmetric input spectrum for Equation (9), the theoretically and observationally motivated (Bieber et al. 1993; Matthaeus et al. 2007; Bruno \& Carbone 2013) three-stage spectral form employed by Engelbrecht \& Burger (2015) is 
used:

$$
S^{2 \mathrm{D}}\left(k_{\perp}\right)=\frac{C_{0} \lambda_{2 \mathrm{D}} \delta B_{2 \mathrm{D}}^{2}}{2 \pi k_{\perp}}\left\{\begin{array}{l}
\left(\frac{\lambda_{2}}{\lambda_{o}}\right)^{-1}\left(\lambda_{o} k_{\perp}\right)^{q},\left|k_{\perp}\right|<\lambda_{o}^{-1} ; \\
\left(\lambda_{2} k_{\perp}\right)^{-1}, \lambda_{o}^{-1} \leqslant\left|k_{\perp}\right|<\lambda_{2}^{-1} ; \\
\left(\lambda_{2} k_{\perp}\right)^{-\nu},\left|k_{\perp}\right| \geqslant \lambda_{2}^{-1} .
\end{array}\right.
$$

With $\lambda_{2}$ as the length scale at which the inertial range (with spectral index $\nu=5 / 3$ ) commences and $\lambda_{o}$ as the length scale at which the energy-containing range begins. An "inner" range, with spectral index $q=3$, is also present to ensure that $S^{2 \mathrm{D}}$ does not assume a finite value at zero wavenumber. Furthermore,

$$
C_{0}=\left[\left(\ln \left(\frac{\lambda_{o}}{\lambda_{2 \mathrm{D}}}\right)+\frac{1}{1+q}+\frac{1}{\nu-1}\right)\right]^{-1} .
$$

The coefficient resulting from the subsequent integration of Equation (8) is similar to those derived for an axisymmetric spectrum with a wavenumber-independent energy-containing range presented by Strauss et al. (2016) and is given by

$$
D_{\perp}(\mu)=\left(\alpha \mu^{2}+\beta\right) \frac{\sqrt{3 \pi} C_{0} \delta B_{2 \mathrm{D}}^{2}}{B_{o} \sqrt{\delta B_{T}^{2}}}\left[f_{1}+f_{2}+f_{3}\right],
$$

where

$$
\begin{aligned}
f_{1}= & \frac{\lambda_{o}}{q}\left(a v \operatorname{erfc}\left(x_{1}\right)-\sqrt{\frac{3}{\pi}} \frac{B_{o} \tau^{-1} \lambda_{\text {out }}}{\sqrt{\delta B_{T}^{2}}} \mathrm{E}_{(q+1) / 2}\left(x_{1}^{2}\right)\right), \\
f_{2}= & \left(\frac{a^{2} \sqrt{\delta B_{T}^{2}} v^{2}}{\sqrt{3 \pi} B_{o} \tau^{-1}}\left[e^{-x_{2}^{2}}-e^{-x_{1}^{2}}\right]\right. \\
& \left.+a v\left[\lambda_{o} \operatorname{erfc}\left(x_{1}\right)-\lambda_{2} \operatorname{erfc}\left(x_{2}\right)\right]\right), \\
f_{3}= & \frac{a v \lambda_{2}}{\nu}\left(\frac { 1 } { \sqrt { 3 } x _ { 2 } ^ { \nu } } \left[\Gamma\left(\frac{\nu+1}{2}\right)\right.\right. \\
& \left.\left.-\Gamma\left(\frac{\nu+1}{2}, x_{2}^{2}\right)\right]+\sqrt{2 \pi} a \operatorname{erfc}\left(x_{2}\right)\right),
\end{aligned}
$$

with

$$
x_{1}=\frac{\sqrt{3}}{a} \frac{B_{o}}{\sqrt{\delta B_{T}^{2}}} \frac{\tau^{-1} \lambda_{o}}{v}, x_{2}=\frac{\sqrt{3}}{a} \frac{B_{o}}{\sqrt{\delta B_{T}^{2}}} \frac{\tau^{-1} \lambda_{2}}{v},
$$

for notational convenience. Note that $E_{(q+1) / 2}$ denotes the exponential integral function, $\Gamma$, with one or two arguments for the gamma or incomplete gamma functions respectively, and erfc, the complimentary error function. The various $f$ terms correspond to contributions from integrating Equation (3), where here, as in the result of Strauss et al. (2016), $f_{2}$ and $f_{3}$, which correspond to integration of the energy-containing and inertial ranges on the 2D spectrum, respectively, provide the predominant contributions to $D_{\perp}$.

The perpendicular diffusion coefficient described by Equation (13) is quite complex and may present some difficulties upon incorporation into a numerical particle transport code. An alternative approach is to evaluate Equation (6) within the standard NLGC approach proposed by Matthaeus et al. (2003). Relatively simple approximate expressions for the pitch-angle isotropic perpendicular diffusion coefficient, $\kappa_{\perp}$, have been derived from this theory by Shalchi et al. (2004) and by Zank et al. (2004), and these in turn have been used with some success in numerical cosmic-ray modulation models (e.g., Moloto et al. 2018). Applying the reasoning outlined in Section 2 and utilizing the Shalchi et al. (2004) approximation for $\kappa_{\perp}$ as rewritten by Burger et al. (2008) for a general ratio of the slab to $2 \mathrm{D}$ variance, results in

$$
D_{\perp}=v \mu^{2}\left[a^{2} \sqrt{3 \pi} \frac{\nu-1}{\nu / 2} \frac{\Gamma(\nu / 2)}{\Gamma((\nu-1) / 2)} \lambda_{2 \mathrm{D}} \frac{\delta B_{2 \mathrm{D}}^{2}}{B_{0}^{2}}\right]^{2 / 3} \lambda_{\|}^{1 / 3} .
$$

It must be noted, however, that the expression used for $\kappa_{\perp}$ in the above was derived assuming a 2D turbulence power spectrum different from that described by Equation (3), in that it has a wavenumber-independent energy-containing range and no "inner" range. Furthermore, the assumption that $\tau^{-1}=v / \lambda_{\|}$ is implicitly made. Lastly, to evaluate Equation (11), choices need to be made for the constants $\alpha$ and $\beta$. Given that the pitchangle isotropic perpendicular diffusion coefficient is related to $D_{\perp}$ by Shalchi (2009, and references therein), so that

$$
\kappa_{\perp}=\frac{1}{2} \int_{-1}^{+1} D_{\perp} d \mu,
$$

so it follows that the integral of the $\mu$-dependent part of Equation (9) should be equal to $2 / 3$ so as to retrieve the pitchangle isotropic result. This implies that $\beta=(1-\alpha) / 3$, where $\beta<1 / 3$ to ensure a positive $\alpha$. As it is unclear at present what choice needs to be made for $\alpha$, in what follows, the present study will somewhat arbitrarily set $\alpha=1 / 3$ for the purpose of discussion.

To compare Equations (13), (14), and the result of evaluating Equation (11) with other theoretical approaches, the pitchangle-scattering-dominated UNLT result presented by Qin \& Shalchi (2014) is considered. This expression is given by

$$
D_{\perp}=\frac{1}{2} a^{2} v \mu^{2} \lambda_{\|} \frac{\delta B_{2 \mathrm{D}}^{2}}{B_{0}^{2}} .
$$

The FLRW perpendicular diffusion coefficient can be derived either from quasilinear theory (QLT; Shalchi 2009), or as a special case of UNLT (Qin \& Shalchi 2014), and is given by

$$
D_{\perp}=a v|\mu| \kappa_{\mathrm{fl}},
$$

where $\kappa_{\mathrm{fl}}$ denotes the field line diffusion coefficient. This quantity is related to the 2D ultrascale (Matthaeus et al. 2007), and for the spectral form given by Equation (3), which is (Strauss et al. 2017)

$$
\kappa_{\mathrm{fl}}^{2}=\frac{1}{2} \frac{\delta B_{2 \mathrm{D}}^{2}}{B_{0}^{2}}\left[\frac{\lambda_{o}^{2}\left(\frac{q+1}{2 q-2}\right)+\lambda_{2}^{2}\left(\frac{1-\nu}{2 \nu+2}\right)}{\frac{1}{q+1}+\frac{1}{\nu-1}+\log \frac{\lambda_{o}}{\lambda_{2}}}\right] .
$$

In order to evaluate and compare Equations (13), (14), (16), and (17), various assumptions need to be made. To do so, solar minimum turbulence conditions corresponding to those assumed by Engelbrecht \& Burger (2015) are assumed, and a value of $\lambda_{o}=0.1 \mathrm{au}$ is assumed (Engelbrecht 2019). Two cases for $\tau^{-1}$ are considered. For the first case where $\tau^{-1}=v / \lambda_{\|}$, the magnetostatic proton quasilinear theory 


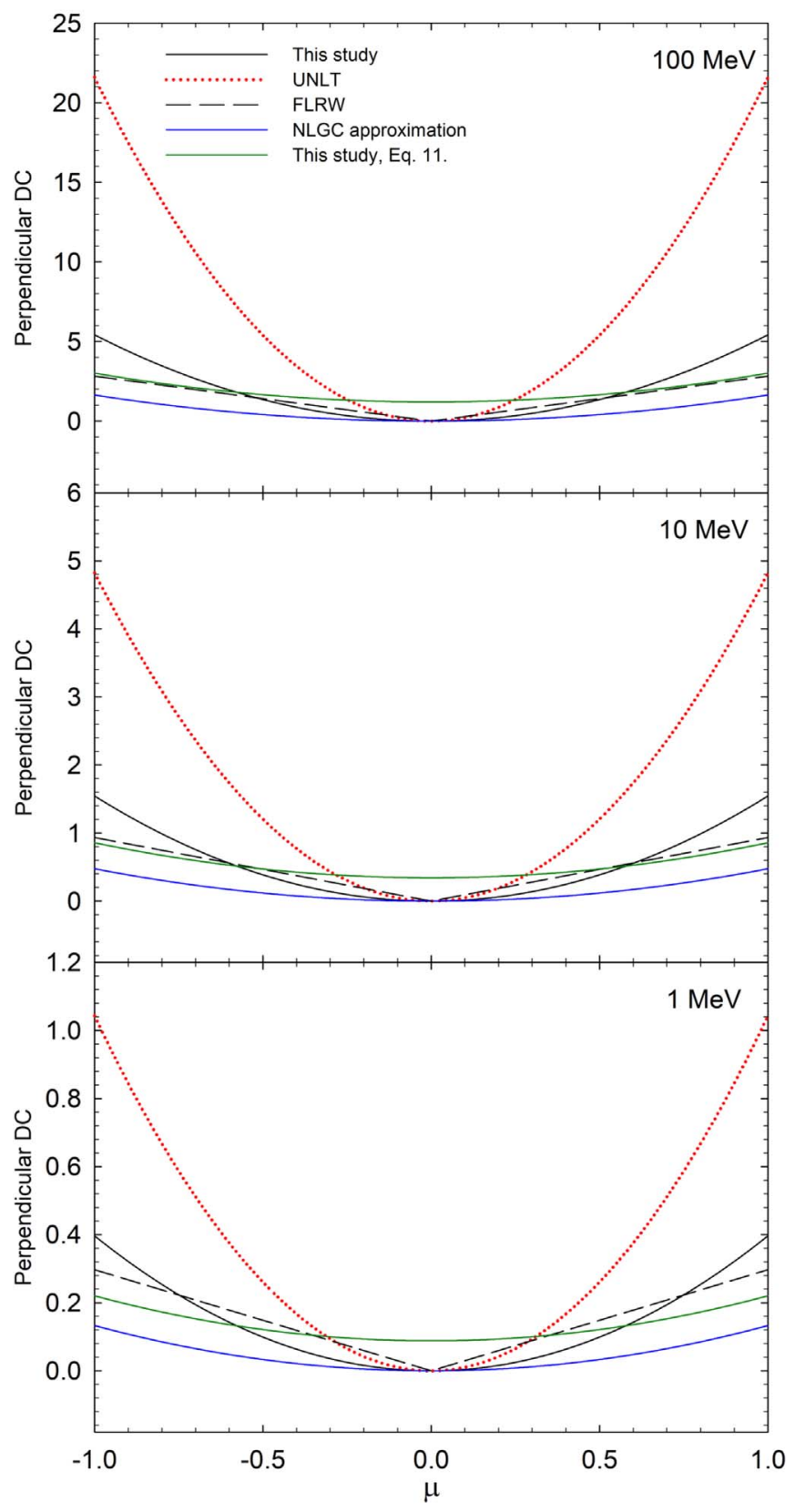

Figure 1. Perpendicular diffusion coefficients calculated from the various theoretical approaches considered in this study at various energies as a function of the pitch-angle cosine $\mu$ for turbulence conditions at Earth, calculated assuming that $\tau^{-1}=v / \lambda_{\|}$. See the text for details.

parallel mean free path employed by Engelbrecht \& Burger (2013a) is used. For the case where $\tau^{-1}=D_{\mu \mu}$ is assumed, the quasilinear theory expression derived for the pitch-angle diffusion coefficient in the presence of magnetostatic slab turbulence by Shalchi et al. (2004) is employed.

The proton perpendicular diffusion coefficients, assuming that $\tau^{-1}=v / \lambda_{\|}$, resulting from these evaluations are shown as a function of $\mu$ for various kinetic energies in Figure 1. For ease of representation, the unit for the perpendicular diffusion coefficient is chosen to be $\mathrm{au}^{2} \cdot \tau_{v s}^{-1}$, where $\tau_{v s}=3.75 \times 10^{5} \mathrm{~s}$ is the time it takes a solar wind proton traveling at $400 \mathrm{~km} . \mathrm{s}^{-1}$ to traverse a distance of $1 \mathrm{au}$. For all energies shown, and for values of $|\mu|$ approaching unity, the UNLT results (red lines) are considerably larger than those for all the other approaches

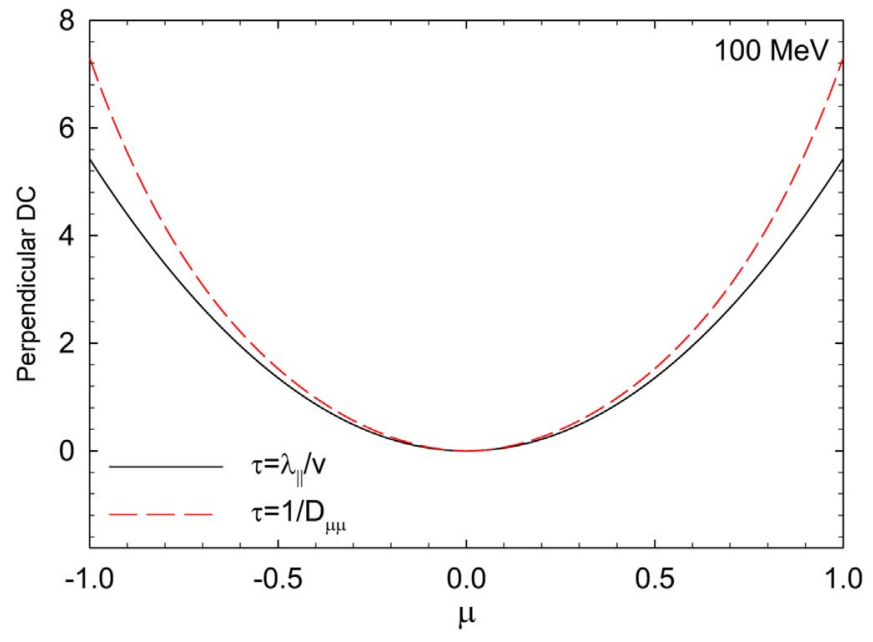

Figure 2. Perpendicular diffusion coefficients calculated according to the approach proposed in this study at $100 \mathrm{MeV}$ as a function of the pitch-angle cosine $\mu$ for turbulence conditions at Earth, assuming that $\tau^{-1}=v / \lambda_{\|}$(black line) and $\tau^{-1}=D_{\mu \mu}$ (red dashed line). See the text for details.

shown, with a considerably steeper increase in the magnitude with an increasing $|\mu|$. The FLRW result, as well as that proposed here, assumes very similar values at small values of $|\mu|$, diverging at larger $|\mu|$ due to their different pitch-angle cosine dependencies. Lastly, the NLGC approximate expression yields, across all energies shown, the smallest values for $D_{\perp}$. Note, though, that this expression was derived for a different form of 2D turbulence spectrum than Equation (3). As expected, all perpendicular diffusion coefficients assume zero values at $\mu=0$. The green lines on Figure 1 correspond to the case where Equation (11) is evaluated along the same lines that led to Equation (13) but assume a $\mu$ autocorrelation function that assumes a finite value at $\mu=0$ (Equation (10)). At all energies shown, this approach leads to modest values for $D_{\perp}$ quite similar to those yielded by the FLRW approach, but, as expected, yields finite values for this quantity at $\mu=0$. Furthermore, a moderate energy dependence can be seen in the values this coefficient assumes at $90^{\circ}$ pitch, which is qualitatively in line with the simulation results of Qin \& Shalchi (2014). The effect of different choices for $\tau^{-1}$ on the perpendicular diffusion coefficients derived in this study is shown in Figure 2, where these $D_{\perp}$ are shown as a function of $\mu$ at an energy of $100 \mathrm{MeV}$. Only a single solution corresponding to the case where $\tau^{-1}=D_{\mu \mu}$ is shown due to the fact that the pitch-angle dependences of both solutions remain similar. The solutions corresponding to $\tau^{-1}=D_{\mu \mu}$ have, as can be seen in the figure, a slightly steeper $\mu$ dependence closer to $|\mu| \leqslant 1$ and remain similar to the results of Qin \& Shalchi (2014). For this case, when $\mu=0$ and $\mu= \pm 1$, Equation (13) does yield indeterminate values, as the magnetostatic QLT expression for the pitch-angle diffusion coefficient employed here is zero at these values of $\mu$. Employing alternative expressions for $D_{\mu \mu}$ that, for example, take into the account the effects of dynamical turbulence (see, e.g., Teufel \& Schlickeiser 2003; Shalchi 2009) and, thus, do not assume zero values would resolve this issue. Overall, assuming that $\tau^{-1}=D_{\mu \mu}$ yields expressions for $D_{\perp}$ comparable with those acquired by the standard approach assuming that $\tau^{-1}=v / \lambda_{\|}$. In what follows, however, the standard assumption is made for the purpose of comparing with the results of existing theories. 


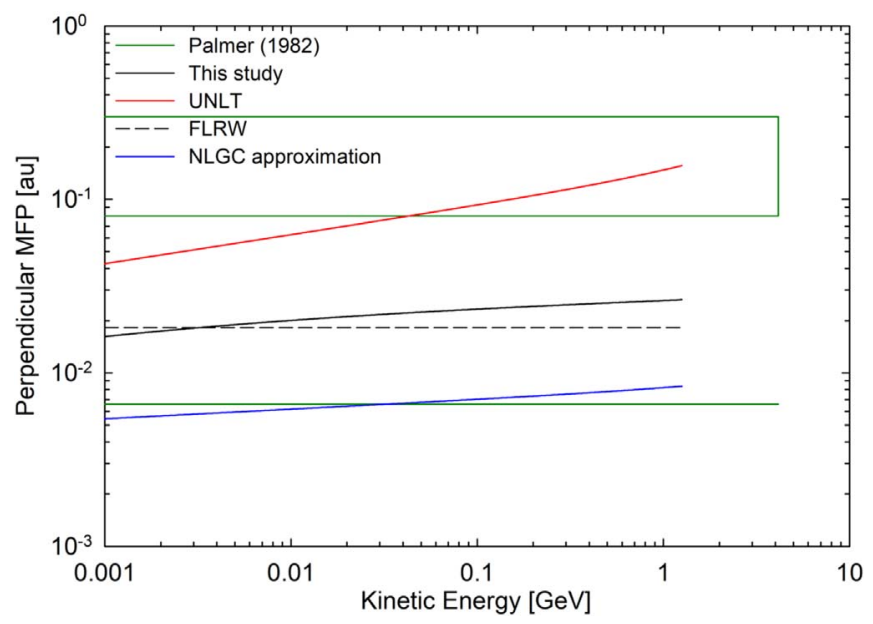

Figure 3. Perpendicular mean free paths corresponding to the various $D_{\perp}$ considered here at various energies as a function of kinetic energy. Note that, by construction, the perpendicular mean free path corresponding to Equation (11) is the same as that corresponding to Equation (13) (solid black line). Palmer (1982) consensus range is indicated by the green lines. See text for details.

If one has an expression for $D_{\perp}$, it is relatively simple to calculate a perpendicular mean free path, as employed in transport studies of pitch-angle isotropic (or nearly so) particles, such as cosmic rays, by averaging $D_{\perp}$ over $\mu$. Such a result can then be compared with the Palmer (1982) consensus range to ascertain whether the perpendicular mean free path so obtained would be compatible with what was assumed in prior transport studies. The results of such a calculation are shown as a function of kinetic energy in Figure 3 along with Palmer consensus range values for the perpendicular as well as the parallel mean free paths. Note that, due to the choices made for parameters $\alpha$ and $\beta$ in the evaluation of Equation (11), the perpendicular mean free path corresponding to this result is equal to that corresponding to Equation (13). The NLGC approximation yields a perpendicular mean free path closest to the Palmer consensus value of the perpendicular mean free path, while the UNLT result provides the largest values, falling within the parallel mean free path consensus range past $\sim 0.05 \mathrm{GeV}$. Not unexpectedly, the result calculated in this study and the FLRW result assume similar values, with the latter displaying a constant energy dependence expected from the Palmer consensus value. It is interesting to note that the FLRW approach, the NLGC approximation, and the approach outlined in this study yield perpendicular mean free path values at 1 au very similar to those yielded by perpendicular mean free path expressions that, when employed in ab initio cosmic-ray modulation codes, yield galactic cosmic-ray proton intensities in reasonable agreement with spacecraft observations at 1 au (see, e.g., Engelbrecht \& Burger 2015; Moloto et al. 2018).

Given the sensitivity of SEP transport model results to assumptions made about $D_{\perp}$, a comparison of the various coefficients discussed here at radial distances closer to the Sun is necessary, given that turbulence conditions vary considerably in the inner heliosphere, which in turn would affect each of the expressions for $D_{\perp}$ considered here. Chhiber et al. (2019) present predictions for the values of various turbulence quantities relevant to this study which they expect to be encountered by the Parker Solar Probe, calculated using a state of the art turbulence transport code (for more detail see, e.g.,
Usmanov et al. 2018). This study shows an approximately $r^{0.5}$ radial dependence for the turbulence correlation scale, and a $\sim r^{-2.5}$ dependence for the fluctuation energy. As such, to get an indication how the various expressions for $D_{\perp}$ evaluated here change under turbulence conditions in the very inner heliosphere, the turnover scales and variances that those expressions are functions of will be scaled in the same way, normalized to solar minimum values at 1 au. Furthermore, a Parker heliospheric magnetic field normalized to a magnitude of $5 \mathrm{nT}$ at Earth is assumed. The $10 \mathrm{MeV}$ results of such an evaluation are shown in Figure 4, as a function of the heliocentric radial distance and $\mu$ in the solar ecliptic plane. All the expressions for $D_{\perp}$ shown in this figure increase with an increasing radial distance, due to the corresponding increase in $\delta B_{2 \mathrm{D}} / B_{o}$ and in the $2 \mathrm{D}$ correlation scale, as modeled in this study. All perpendicular diffusion coefficients also displayed flatter $\mu$ dependences at smaller radial distances. In the top two panels of this figure, Equation (13) and the UNLT result are compared on the same scale. At the smallest radial distances, both results are similar, with the UNLT result assuming considerably larger values approaching 1 au and a steeper increase as $|\mu|$ approaches unity. The bottom four panels of Figure 4 compare the results of evaluating Equation (13) with the remaining theoretical approaches considered here on the same scale. At the smallest radial distance considered here, FLRW and the approach proposed here yield very similar diffusion coefficients. The FLRW result also displays an increasingly steep increase as $|\mu|$ approaches 1, as expected, which remains at values smaller than those yielded by Equation (13). Equations (13) and (11), as well as the NLGC approximation result, behave in a similar manner as they increase in magnitude with the radial distance and closer to Earth, they display correspondingly steeper increases as $|\mu|$ approaches unity. Equation (11) yields a relatively flat (as a function of $\mu$ ) perpendicular diffusion coefficient at the smallest radial distance considered here, reflecting the behavior of Equation (13), but never assumes zero values, as expected. Of all the perpendicular diffusion coefficients considered here, the NLGC approximation yields the smallest values for $D_{\perp}$.

\section{Discussion and Conclusions}

A fuller understanding of the perpendicular diffusion coefficient is vital to our understanding of the transport of charged particles in the heliosphere. More particularly, this quantity has been demonstrated to be of particular importance to the study of the transport of SEPs and their acceleration (see, e.g., the discussion by Zank et al. 2004). This study proposes a new approach to calculating $D_{\perp}$ that is different to what has been used in previous studies in that it employs the random ballistic decorrelation interpretation of the NLGC theory proposed by Ruffolo et al. (2012) and directly attempts to model the pitch-angle cosine autocorrelation function for moderate levels of turbulence, as motivated by the simulations of Casse et al. (2002) and Qin \& Shalchi (2009). An expression for $D_{\perp}$ is derived using this approach, assuming an observationally motivated form for the $2 \mathrm{D}$ turbulence power spectrum. Qualitatively, for $\mu \neq 0$, the pitch-angle cosine dependence of this coefficient, as well as its behavior at higher energies, is in agreement with the simulation results of Qin \& Shalchi (2014). An analytical approximation, derived under the same assumptions for the pitch-angle cosine autocorrelation function and based on the NLGC result of Shalchi et al. (2004), 

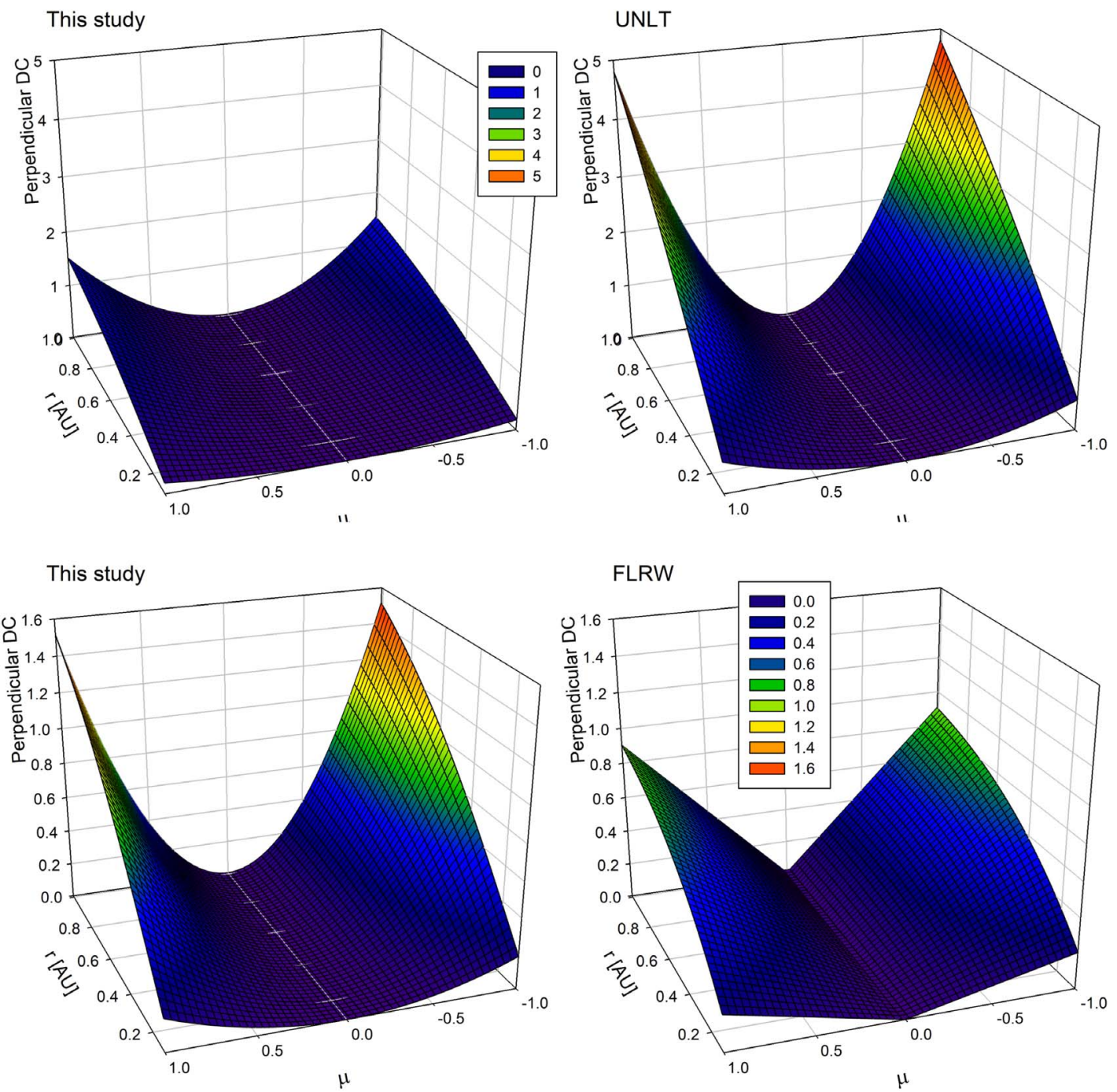

This study, Eq. 11.

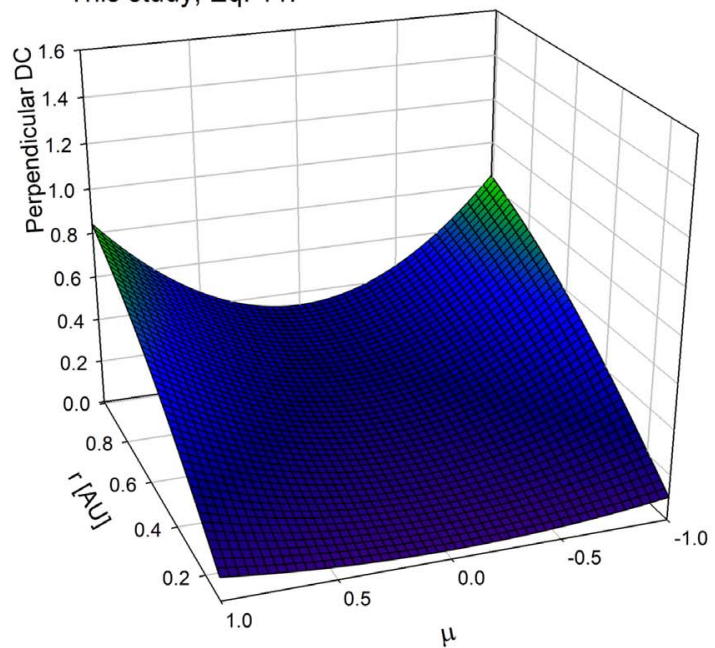

NLGC approximation

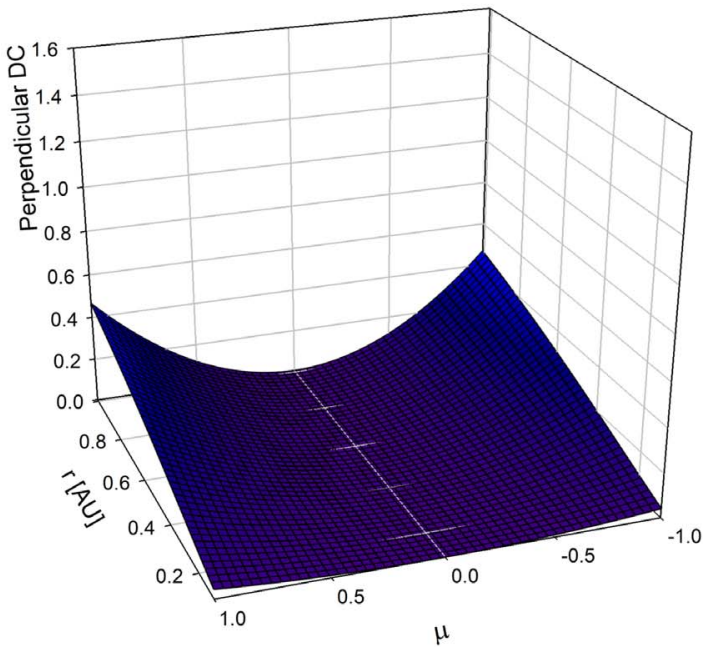

Figure 4. Comparison of $10 \mathrm{MeV}$ perpendicular diffusion coefficients calculated using the approach proposed in this study (left panels) with those calculated using the UNLT approach (top right panel), the FLRW approach (middle left panel), the NLGC approximation to the approach taken in this study (bottom right panel), and the approach taken in Equation (11), as a function of the radial distance and pitch-angle cosine, assuming simple radial scalings for relevant turbulence quantities in the solar ecliptic plane. The unit chosen for $D_{\perp}$ is au. $\tau_{\mathrm{sw}}^{-1}$. See the text for details. Note also that, for the purposes of comparison, scales in the top two and bottom four panels are chosen differently. 
is also presented. This result, however, yields values for $D_{\perp}$ that are considerably smaller than the other approaches considered in this study.

It is a standard approach in various scattering theories considered here to decorrelate velocity autocorrelation functions exponentially with time, using some timescale, $\tau^{-1}=v / \lambda_{\|}$. In the present study, whether this decorrelation timescale could not rather be modeled using the pitch-angle diffusion coefficient was investigated, so that $\tau^{-1}=D_{\mu \mu}$. The latter assumption, when used in the approach to calculating $D_{\perp}$ presented here, was found to not greatly differ from results calculated assuming that $\tau$ is a function of the parallel mean free path. The analysis presented by Adhikari et al. (2019) of the Zank et al. $(2012,2017)$ turbulence transport models close to the Alfvén surface lead these authors to conclude that slab modes may vanish at this surface, while quasi-2D modes do not. Given that most modeling approaches assume some QLT expression for the parallel mean free path, this could make the assumption that $\tau^{-1}=v / \lambda_{\|}$somewhat problematic. Assuming that $\tau^{-1}=D_{\mu \mu}$ may provide a way around that problem, this quantity has been shown to assume finite values for purely $2 \mathrm{D}$ turbulence (see, e.g., Shalchi 2009) and, thus, could prove useful in SEP transport modeling endeavors.

Furthermore, an alternate functional form for the pitch-angle cosine autocorrelation function is speculated upon, which in the approach outlined here leads to $D_{\perp} \sim \alpha \mu^{2}+\beta$. The resulting coefficient agrees qualitatively with numerical simulations in that this coefficient displays a similar energy dependence to the simulation results of Qin \& Shalchi (2014) in terms of the energy dependence of the value of $D_{\perp}$ at $\mu=0$, as well as the relatively flat increase of this coefficient as $|\mu|$ approaches unity at larger energies. However, the form chosen for the pitchangle cosine autocorrelation function is not motivated at all by existing numerical test particle simulations, and it remains to be seen whether future simulations of this quantity would yield the functional form of $\langle\mu(0) \mu(\tau)\rangle \sim\left(\alpha \mu^{2}+\beta\right) \exp (-t / \tau)$ or not.

The perpendicular diffusion coefficients derived using the above approaches were then compared to the FLRW and UNLT results presented by Qin \& Shalchi (2014), assuming observationally motivated solar minimum values for turbulence quantities at Earth. The various approaches yield significantly different perpendicular diffusion coefficients, whether in magnitude or in pitch-angle dependence. The UNLT approach consistently lead to larger values for $D_{\perp}$ than the other results considered. This, however, is not necessarily a disadvantage, as this feature may help resolve the issue of too-fast cross-field SEP propagation without assuming non-diffusive perpendicular transport (see, however, the discussion by Laitinen et al. 2013 , 2018). However, when perpendicular mean free paths are calculated using the various theoretical expressions for $D_{\perp}$ and compared with Palmer (1982) consensus values for this quantity, the UNLT results are considerably larger than the FLRW results and those calculated using the approach outlined in this study, which fall in the range expected from galactic cosmic-ray modulation studies. The proposed approximate solution falls closest to the Palmer consensus range, but it should be noted that this range is constructed using results reported for diffusion coefficients by many numerical charged particle transport studies and would be subject to the limitations imposed by the various assumptions made in these studies (see, e.g., Palmer 1982; Bieber et al. 1994, and references therein). Further comparisons of the perpendicular diffusion coefficients yielded by these various theories were made within 1 au, assuming simple radial scalings for relevant turbulence quantities, as motivated by the turbulence transport model results presented by Chhiber et al. (2019). All the theoretical approaches were found to yield considerably different predictions for $D_{\perp}$, where all were quite sensitive to the behavior of turbulence quantities in the very inner heliosphere.

Given the sensitivity of SEP transport models to assumptions made about the perpendicular diffusion coefficient, the differences in the results yielded by the various theoretical approaches considered here should have significant effects on SEP transport modeling efforts. More comparative numerical test particle simulations need to be done for simulated turbulence conditions similar to those encountered well within $1 \mathrm{au}$ in order for a proper consensus to be reached as to which approach is best taken when modeling SEP transport. Furthermore, given the sensitivity of the theoretical expressions to the behavior of turbulence quantities, comparative studies of observed SEP events using numerical transport codes linked to turbulence transport models, such as those of Wiengarten et al. (2016), Adhikari et al. (2017), or Chhiber et al. (2019), would also give more insight into this important quantity.

N.E.E. would like to thank R.D. Strauss and P. Els for valuable discussions on the various subjects of this study, as well as an anonymous referee for their careful review of this paper.

\section{ORCID iDs}

N. Eugene Engelbrecht (iD https://orcid.org/0000-00033659-7956

\section{References}

Adhikari, L., Zank, G. P., Telloni, D., et al. 2017, ApJ, 851, 117

Adhikari, L., Zank, G. P., \& Zhao, L.-L. 2019, ApJ, 876, 26

Agueda, N., \& Vainio, R. 2013, JSWSC, 3, A10

Bain, N. H., Kontar, E. P., \& MacKinnon, A. L. 2011, A\&A, 535, A18

Bieber, J. W., Chen, J., Matthaeus, W. H., Smith, C. W., \& Pomerantz, M. A. 1993, JGR, 98, 3585

Bieber, J. W., Matthaeus, W. H., Smith, C. W., et al. 1994, ApJ, 420, 294

Bruno, R., \& Carbone, V. 2013, LRSP, 10, 2

Burger, R. A., Krüger, T. P. J., Hitge, M., \& Engelbrecht, N. E. 2008, ApJ, 674, 511

Burlaga, L. F., Florinski, V., \& Ness, N. F. 2018, ApJ, 854, 20

Casse, F., Lemoine, M., \& Pelletier, G. 2002, PhRvD, 65, 023002

Chhiber, R., Usmanov, A. V., Matthaeus, W. H., Parashar, T. N., \& Goldstein, M. L. 2017, ApJS, 230, 21

Chhiber, R., Usmanov, A. V., Matthaeus, W. H., Parashar, T. N., \& Goldstein, M. L. 2019, ApJS, 242, 12

Dresing, N., Gomez-Herrero, R., Klassen, A., et al. 2012, SoPh, 281, 281

Droge, W., Kartavykh, Y. Y., Klecker, B., \& Kovaltsov, G. A. 2010, ApJ, 709, 912

Engelbrecht, N. E. 2019, ApJ, 872, 124

Engelbrecht, N. E., \& Burger, R. A. 2013a, ApJ, 772, 46

Engelbrecht, N. E., \& Burger, R. A. 2013b, ApJ, 779, 158

Engelbrecht, N. E., \& Burger, R. A. 2015, ApJ, 814, 152

Engelbrecht, N. E., Strauss, R. D., le Roux, J. A., \& Burger, R. A. 2017, ApJ, 841, 107

Fraschetti, F. 2016, ASTRAP, 2, 63

Fraschetti, F., \& Jokipii, J. R. 2011, ApJ, 734, 83

Gammon, M., \& Shalchi, A. 2017, ApJ, 847, 118

Green, M. S. 1951, JChPh, 19, 1036

He, H-Q. 2015, ApJ, 814, 2

He, H-Q., \& Wan, W. 2012, ApJ, 747, 38

Jokipii, J. R. 1966, ApJ, 146, 480

Karimabadi, H., Krauss-Varban, D., \& Terasawa, T. 1992, JGR, 97, 13853

Kubo, R. 1957, JPSJ, 12, 570 
Laitinen, T., \& Dalla, S. 2017, ApJ, 834, 127

Laitinen, T., Dalla, S., \& Marsh, M. S. 2013, ApJL, 773, L29

Laitinen, T., Effenberger, F., Kopp, A., \& Dalla, S. 2018, JSWSC, 8, A13

Lasuik, J., \& Shalchi, A. 2018, AdSpR, 61, 2827

Matthaeus, W. H., Bieber, J. W., Ruffolo, D., Chuychai, P., \& Minnie, J. 2007, ApJ, 667, 956

Matthaeus, W. H., Qin, G., Bieber, J. W., \& Zank, G. P. 2003, ApJL, 590, L53

McKibben, R. B., Lopate, C., \& Zhang, M. 2001, SSRv, 97, 257

Minnie, J., Bieber, J. W., Matthaeus, W. H., \& Burger, R. A. 2007a, ApJ, 663, 1049

Minnie, J., Bieber, J. W., Matthaeus, W. H., \& Burger, R. A. 2007b, ApJ, 670,1149

Moloto, K. D., Engelbrecht, N. E., \& Burger, R. A. 2018, ApJ, 859, 107

Palmer, I. D. 1982, RvGSP, 20, 335

Qin, G., Matthaeus, W. H., \& Bieber, J. W. 2002, ApJL, 578, L117

Qin, G., \& Shalchi, A. 2009, ApJ, 701, 61

Qin, G., \& Shalchi, A. 2014, ApPhR, 2, 1

Reames, D. V. 1999, SSRv, 90, 413

Riordan, J. D., \& Pe'er, A. 2019, ApJ, 873, 1

Rossi, B., \& Olbert, S. 1970, Introduction to the Physics of Space (New York: McGraw-Hill)

Ruffolo, D., Pianpanit, T., Matthaeus, W. H., \& Chuychai, P. 2012, ApJL, 747, L34

Schwadron, N. A., Blake, J. B., Case, A. W., et al. 2014, SpWea, 12, 622

Shalchi, A. 2006, A\&A, 453, L43
Shalchi, A. 2009, Nonlinear Cosmic Ray Diffusion Theories (Berlin: Springer) Shalchi, A. 2010, ApJL, 720, L127

Shalchi, A., Biever, J. W., \& Matthaeus, W. H. 2004, ApJ, 604, 675

Skilling, J. 1971, ApJ, 170, 265

Strauss, R. D., Dresing, N., \& Engelbrecht, N. E. 2017, ApJ, 837, 43

Strauss, R. D., \& Fichtner, H. 2014, A\&A, 572, L3

Strauss, R. D., \& Fichtner, H. 2015, ApJ, 801, 29

Strauss, R. D., \& le Roux, J. A. 2019, ApJ, 872, 2

Strauss, R. D., le Roux, J. A., Engelbrecht, N. E., Ruffolo, D., \& Dunzlaff, P. 2016, ApJ, 825, 43

Tautz, R. C., \& Shalchi, A. 2010, PhPl, 17, 122313

Tautz, R. C., \& Shalchi, A. 2012, ApJ, 744, 125

Taylor, G. I. 1922, Proc. Lond. Math. Soc., 20, 196

Teufel, A., \& Schlickeiser, R. 2003, A\&A, 397, 15

Usmanov, A. V., Matthaeus, W. H., Goldstein, M. L., \& Chhiber, R. 2018, ApJ, 865, 1

Wang, J. F., Qin, G., Ma, Q. M., Song, T., \& Yuan, S. B. 2017, ApJ, 845, 112

Webb, G. M., Kota, J., Zank, G. P., \& Lu, J. Y. 2001, Proc. ICRC 2001, 3326

Wiengarten, T., Oughton, S., Engelbrecht, N. E., et al. 2016, ApJ, 833, 17

Zank, G. P., Adhikari, L., Hunana, P., et al. 2017, ApJ, 835, 147

Zank, G. P., Adhikari, L., Hunana, P., et al. 2018, ApJ, 854, 32

Zank, G. P., Dosch, A., Hunana, P., et al. 2012, ApJ, 745, 35

Zank, G. P., Li, G., Florinski, V., et al. 2004, JGR, 109, A04107

Zhang, M., Jokipii, J. R., \& McKibben, R. B. 2003, ApJ, 595, 493

Zhang, M., Qin, G., \& Rassoul, H. 2009, ApJ, 692, 109 